(C) 2011 IEEE. Personal use of this material is permitted. Permission from IEEE must be obtained for all other uses, in any current or future media, including reprinting/republishing this material for advertising or promotional purposes, creating new collective works, for resale or redistribution to servers or lists, or reuse of any copyrighted component of this work in other works.

Published in: Jiménez, J.; Iglesias, A.M.; López, J.F.; Hernández, J.; Ruiz, B., Tablet PC and Head Mounted Display for live closed captioning in education, 2011 IEEE International Conference on Consumer Electronics (ICCE), pp. 885-886, 9-12 January 2011.

Doi: http://dx.doi.org/10.1109/ICCE.2011.5722919 


\title{
Tablet PC and Head Mounted Display for Live Closed Captioning in Education
}

\author{
Javier Jiménez, Ana M. Iglesias, Juan F. López, Julián Hernández and Belén Ruiz
}

\begin{abstract}
Automatic Speech Recognition is a powerful tool for inclusive education. Captioning helps disabled students but distract ot her students. We propose and evaluate the use of Tablets PC and Head Mounted Displays for live closed captioning.
\end{abstract}

Index Terms - Automatic Sp eech Recognition, Closed captioning, Inclusive education, Displays.

\section{INTRODUCTION}

In inclusive education context, speech technologies have become as the best tool to enhance students' learning. Particularly, Automatic Speech Recognition (ASR) provides a cost effective solution for any student with disability when they cannot easily follow the speech or take notes (e.g. deaf, hard of hearing, blind or physically impaired students).

However, we have to ensure that live captioning does not disturb students who do not need it during the class. This is why closed captioning is necessary in education [1] (and other fields such as cinem a). Hence, it is im portant to distinguish closed captioning from open captioning.

In this paper, we highlight the im portance of live closed captioning in the inclusi ve education. Closed captioning provides individual captions to those users who need or want it and avoids distraction of other students. W e present a proposal for live closed captioning in education with two emerging devices, Tablet PC and Head Mounted Displays (HMD). And we carried out a user evaluation using a Tablet $\mathrm{PC}$ and a HMD to read captions com pared to reading captions from a big screen (open captioning).

\section{SPEech TeChNOLOGIES IN EdUCATION}

Researchers around the world are working on removing educational barriers in education through speech technologies, especially Automatic Speech Recognition (ASR). The common idea is that, the ASR software provides a real time transcription of teacher's speech.

The most relevant and ol dest work using speech technologies in education is the Liberated Learning Consortium [2]. They developed ViaScribe where they also considered the use of personal di splays and real tim e ASR errors edition. There are other im portant and sim ilar works. With VUST (Villanova University Speech Transcription), authors reported that accuracy strongly depends on speaking behavior [3]. LECTRA syst em (Classroom Lecture Transcriptions in European Portuguese) dem onstrated the utility of speech technologies on recorded $\mathrm{m}$ edia, like videos or audio files [4] . Another interesting project is the Spoken Lecture Processing Project which was initially designed for video indexing [5].

\section{A. Closed Captioning vs Open Captioning}

Closed captioning refers the fact of displaying captions only to those people who need or want it. Contrary to closed, it is open captioning where captions are di splayed to the whole audience regardless of whether each person wants to read it.

On the one hand, open captioning is cheaper because it is only necessary a screen where projecting the captions on for all students. Besides, depending on captions location, this method may be the best way to read because both video and captioning are in the same focal plane. On the other hand, the main disadvantage of open captioni $\mathrm{ng}$ is that non disabled students may be distracted.

Closed captioning is well known in television but it is not so well in education. W hile in television is as easy as switching on/off captioning, in education it is needed a device for each student who want to read captioning. Therefore, it is more expensive compared to open captioning. However, it is the only way to avoid students without disabilities to get distracted by live captioning in education. Besides, closed captioning allows students to configure individually some captions parameters (i.e. size, color, speed and font). Apart from costs, a priori im portant problem in closed captioning is that there are two inform ation sources, the teacher plane (with slides, blackboard, etc.) and the individual device plane (where reading the captioning). Focusing on two different planes may be hard and tiring for students.

\section{A Proposal for Live Closed Captioning in EDUCATION}

The proposal is fram ed within the APEINTA system [6]. Inside the classroom, APEINTA provides a transcription of what the teacher is saying in real tim e. This transcription is displayed to disabled students to assist them in note taking. Outside the classroom, the use of APEINTA provides automatically synchronized educational resources (i.e. slides, notes, video or audio) and allows media mining indexing.

Considering the closed captioning problem $\mathrm{s}$, we implemented new clients in a Ta blet PC and in a HMD where students can read captions ${ }^{1}$.

The main objective of this work is to evaluate the potential and utility of two em erging t echnologies as device for live closed captioning in education.

\footnotetext{
${ }^{1}$ As Tablet PC we use an iPad and as HMD model T3-F by Teleglass.
} 


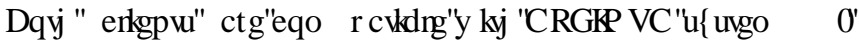

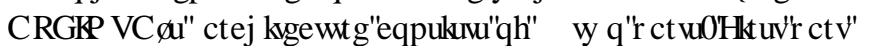

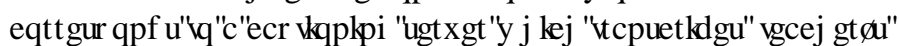

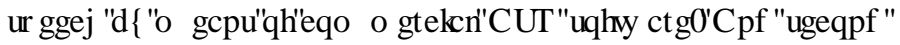
SDWFRUHSRQGVURIQAMGXDO FOHQWGHIFHVZ KHHRQQ]

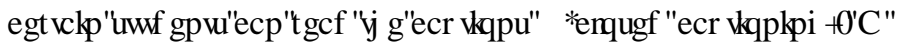

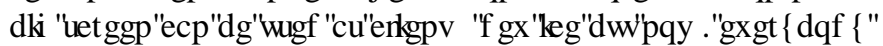
FDQUHDOWWHFDSWRQMURSHQFDSWRQQD

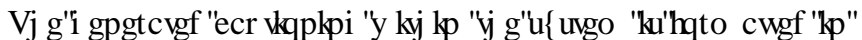

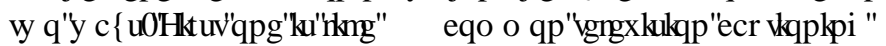

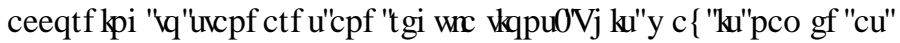

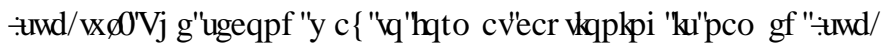

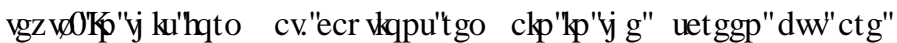

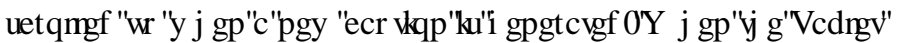

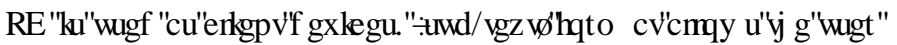

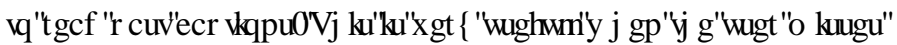
VRP HIIQRUP DNRQD

\section{, $9 \square(9 \$ / 8 \$ 7,21$}

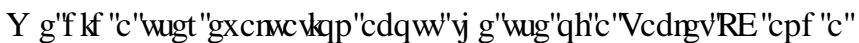

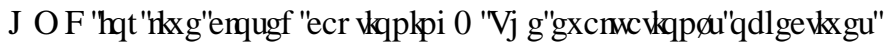

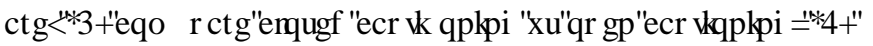

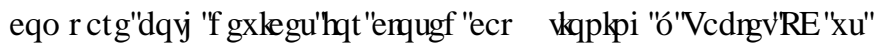

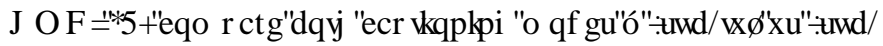

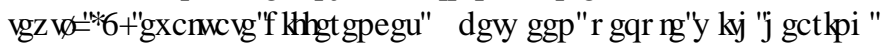
GUEICWWYYVSHRSGIZ IKRXXWW

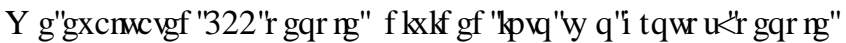

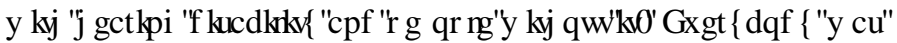

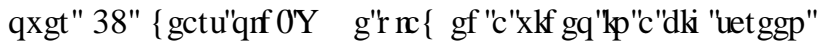

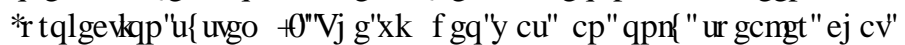
DERXWWJ HQHOOURSIFW\%RUXDXGRIDCGFDSWRQVODQJ XD HZ HH

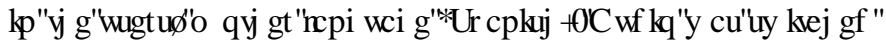

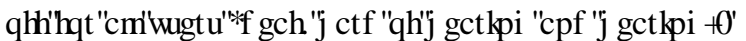

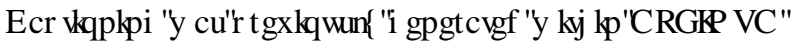

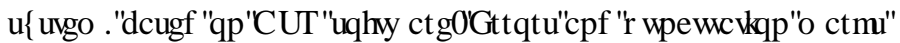

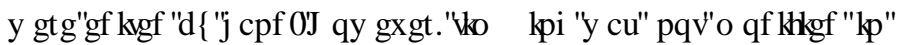

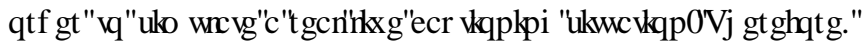

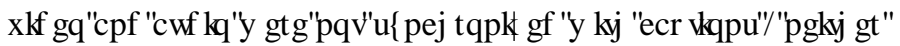
FRRVHGQRURSHQI7 KHHILVIDGHD IGXHIMRS 65 [SLRFHMU

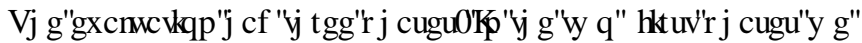

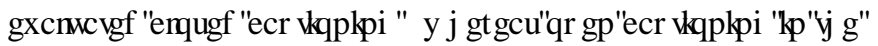

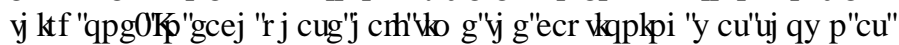

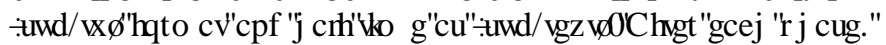

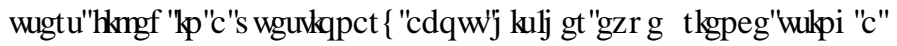
ILYHSRIQW INHWWEDOHI7 KH WORTGGIDUOQNQ IRILXHEHW GHMFHIIRUCOYHFDSWRQID TFCRVHGDQGRSHQW

\section{$9 \square 5(68 / 76$}

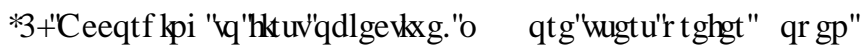

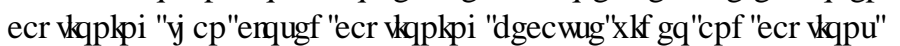

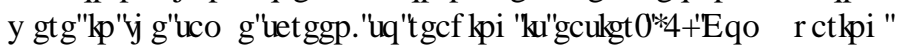

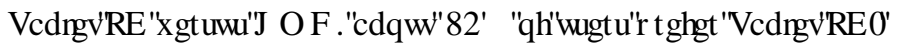
, WKDVIIDEIJ J HUVFUHQIDQGIIRQWFDQEHIP RUHUHODECII5 HDAQI

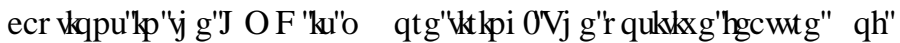

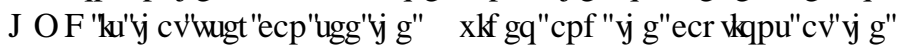

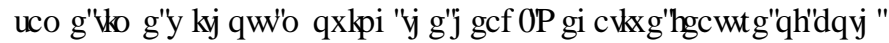

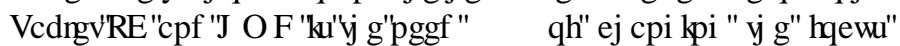

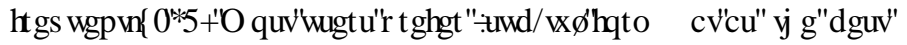

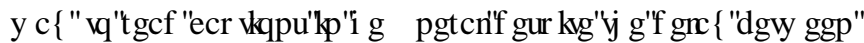

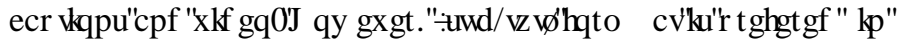
WAH7 DECOHBB \& EHFDXVHMLHXVHUFDQELRZ VHIMHI Z Z KROD

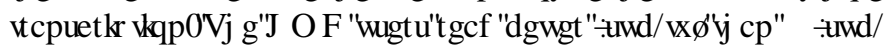

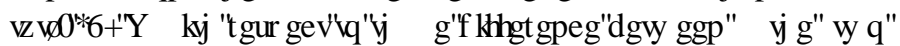

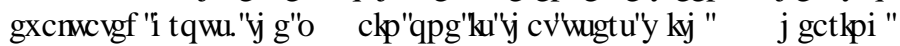

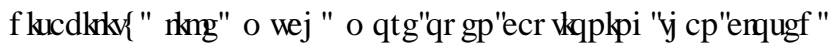

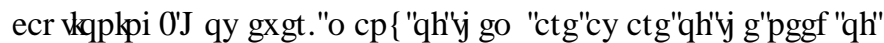
FGRVHGIFDSWRQQD ITX WAP VIIQHEXFDARQW

\section{$9, \square \& 21 \& / 86,21$}

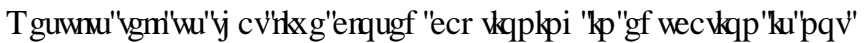

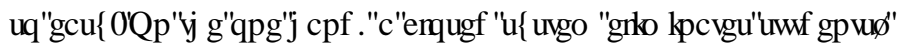

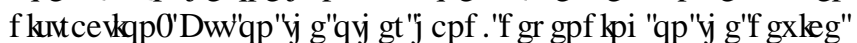

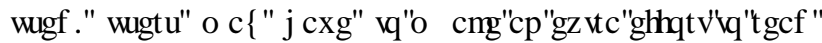
FDSWRQQD WOQGQRKP LWILQRLP DWRQW

2 SHQFDSWRQQ GLVWAHEHWZ D IXIUDOUWDONP HFDSWRQV

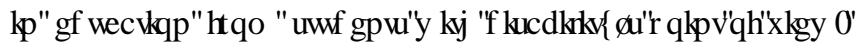

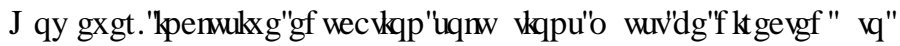

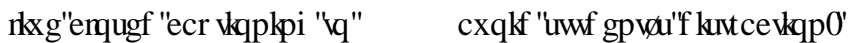

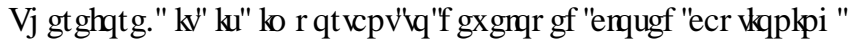

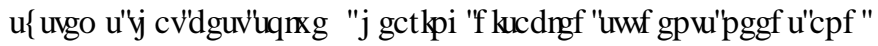

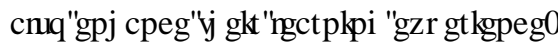

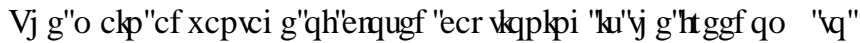

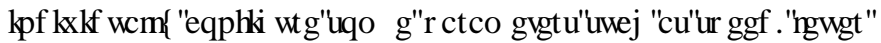

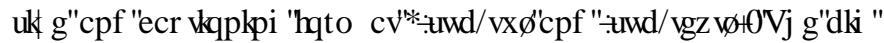

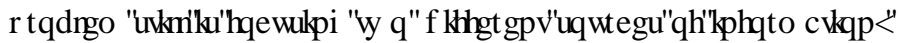

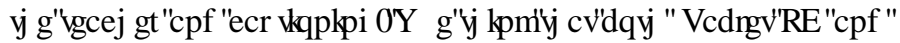

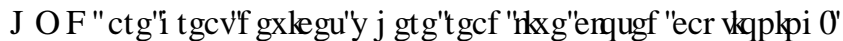

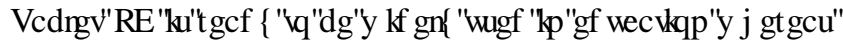

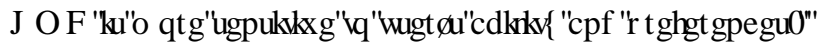

$$
5 \text { ( ) }(5 \text { ( } 1 \&(6
$$

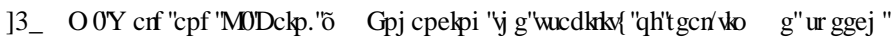

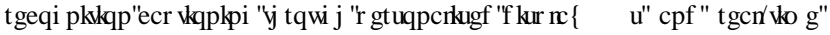

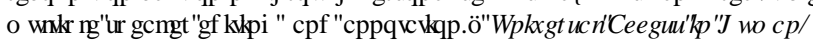

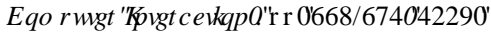

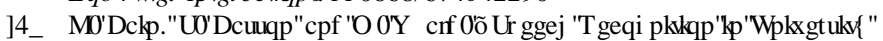

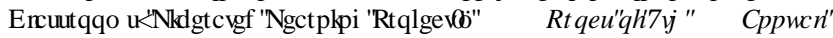

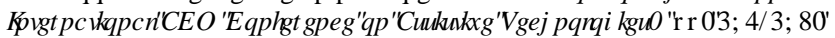
प्र०ण

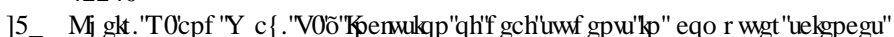

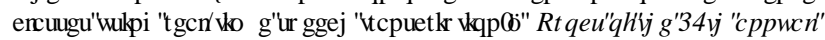

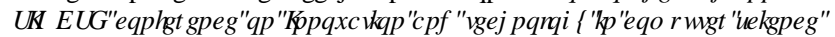

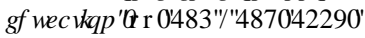

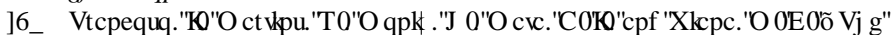

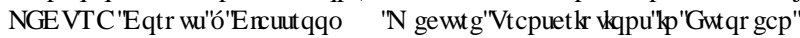

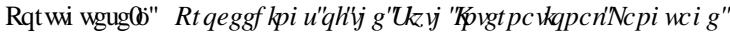
5HRXLFHWDQE( YDODWRQIIUTUT

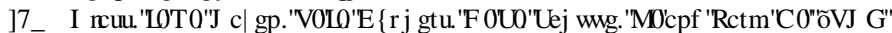

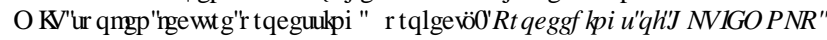

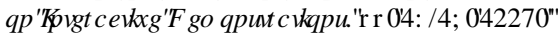

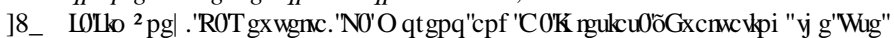

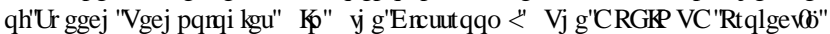

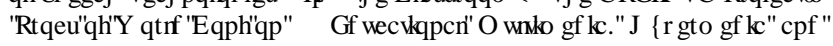

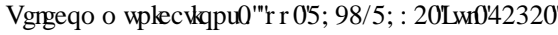

